\title{
The determination of high-resolution spatio-temporal glacier motion fields from time-lapse sequences
}

\author{
Ellen Schwalbe and Hans-Gerd Maas \\ Institute of Photogrammetry and Remote sensing, Technische Universität Dresden, 01069 Dresden, Germany \\ Correspondence: Ellen Schwalbe (ellen.schwalbe@tu-dresden.de)
}

Received: 10 May 2017 - Discussion started: 30 May 2017

Revised: 2 November 2017 - Accepted: 13 November 2017 - Published: 21 December 2017

\begin{abstract}
This paper presents a comprehensive method for the determination of glacier surface motion vector fields at high spatial and temporal resolution. These vector fields can be derived from monocular terrestrial camera image sequences and are a valuable data source for glaciological analysis of the motion behaviour of glaciers. The measurement concepts for the acquisition of image sequences are presented, and an automated monoscopic image sequence processing chain is developed. Motion vector fields can be derived with high precision by applying automatic subpixel-accuracy image matching techniques on grey value patterns in the image sequences. Well-established matching techniques have been adapted to the special characteristics of the glacier data in order to achieve high reliability in automatic image sequence processing, including the handling of moving shadows as well as motion effects induced by small instabilities in the camera set-up. Suitable geo-referencing techniques were developed to transform image measurements into a reference coordinate system.

The result of monoscopic image sequence analysis is a dense raster of glacier surface point trajectories for each image sequence. Each translation vector component in these trajectories can be determined with an accuracy of a few centimetres for points at a distance of several kilometres from the camera. Extensive practical validation experiments have shown that motion vector and trajectory fields derived from monocular image sequences can be used for the determination of high-resolution velocity fields of glaciers, including the analysis of tidal effects on glacier movement, the investigation of a glacier's motion behaviour during calving events, the determination of the position and migration of the grounding line and the detection of subglacial channels during glacier lake outburst floods.
\end{abstract}

\section{Introduction}

For almost a century, terrestrial photogrammetry has been an important measurement method for glaciology research. Early basics for the use of photogrammetric methods for velocity measurements on glaciers were provided by Finsterwalder (1931) and Pillewizer (1938). For glaciers in the Pamir, the Himalaya, Norway and the Alps, velocity profiles were derived by repeated measurements. With the advance of the technical development of the cameras, the acquisition intervals for the photogrammetric measurements became shorter. For the investigation of the short-term motion behaviour of glaciers, automatic analogue cameras were used from the 1970s onwards (e.g. Flotron, 1973). Further examples of the use of automatic cameras are the photogrammet- ric time-lapse measurements on Columbia Glacier (Alaska) by Krimmel and Rasmussen (1986), which were conducted with a temporal resolution of three images per day, and the investigations of Variegated Glacier (Alaska) by Harrison et al. (1986).

With the appearance of digital cameras and the rapid development of their sensors, photogrammetric tools have been available since the end of the 20th century which allow for an automatic acquisition of data sets with not only high spatial but also high temporal resolution. Because of further technical improvements of digital cameras and developments in automatic image matching techniques, terrestrial image sequence analysis has become a valuable tool for the investigation of glaciers during the last 20 years. Phenomena with short-term movement variations requiring a temporal reso- 
lution of hours (tidal influence on the glacier movement) or even minutes (calving events) can now be investigated efficiently. Early applications of photogrammetry-based methods for the determination of glacier velocities from terrestrial digital time-lapse measurements were published by Maas et al. (2006) and Ahn and Box (2010). Since then, time-lapse measurements have increasingly been used for glacier motion analysis (e.g. Eiken and Sund, 2012; Rivera et al., 2012; Danielson and Sharp, 2013).

Beyond glaciology applications, time-lapse imagery is also of great interest for various geomorphologic investigations.

Walter (2011) recorded an image sequence of the volcano dome growth of Mount St. Helens in 2006 and applied crosscorrelation techniques to the images in order to derive motion patterns of the volcano's spine. These allowed for a spatial and temporal investigation of the dome deformations. Regarding the investigation of volcanoes, time-lapse images are also of great value for the observation and measurement of active lava flows (e.g. James and Robson, 2014). Parajka et al. (2012), for example, used time-lapse images recorded with an hourly time interval to observe the snow cover in small catchments. Travelletti et al. (2012) evaluated the suitability of terrestrial time-lapse measurements for permanent landslide monitoring. For this purpose a landslide area was observed with a 4-day time interval and displacement rates were derived applying cross-correlation matching to the image series. Matsuoka et al. (2014) conducted a 5-year investigation with time-lapse images to observe soil movement due to frost creep and heave on alpine slopes, taking images every $4 \mathrm{~h}$. To monitor and analyse large wood loads in rivers, Kramer and Wohl (2014) recorded images every minute and manually analysed them regarding the presence or absence of wood. Nichols et al. (2016) used time-lapse images to qualify gully erosion. They identified the subsurface erosion as a cause for gully headwall retreat. Therefore, the time-lapse measurements were considered as an important supplement besides a topographic survey with a total station and lidar. Stumpf et al. (2016) even used time-lapse measurements to determine river discharge.

The simple use of time-lapse cameras as a visual observation tool may already be a great help for environmental investigations. However, beyond that, they have the potential to also deliver precise measurements with high temporal and spatial resolution when applying appropriate photogrammetric image sequence processing techniques. The relevant tools have been provided by, e.g., James et al. (2016) or Messerli and Grinsted (2015). In this paper we want to present a detailed methodological concept for the determination of motion vector fields from terrestrial time-lapse image sequences. In comparison to previous methods, the presented approach also addresses specific problems such as camera motion effects and shadow-induced motion artefacts as well as various aspects of geo-referencing from a photogrammetric perspective. We provide methodological solutions for these aspects in order to exhaust the potential of terrestrial time-lapse imagery regarding both accuracy and reliability. The presented approach has been developed in the course of several glacier measurement projects between 2004 and 2015 (e.g. Dietrich et al., 2007; Rosenau et al., 2013; Schwalbe et al., 2016). This paper has the aim of integrating the experiences gained from these projects. While the method is thus primarily designed for the observation of glaciers, it can also easily be adapted to other environmental motion analysis tasks.

The main part of the paper will be about methodological issues of photogrammetric image sequence analyses (Sect. 2). After introducing the basic concept of the method (Sect. 2.1), several aspects regarding the measurement set-up for time-lapse image acquisition will be discussed (Sect. 2.2). The analysis of the image sequences consists of two main parts. The first one comprises the determination of motion vectors from the images (Sect. 2.3) and the second one the scaling and geo-referencing of the measurement values (Sect. 2.4). The methodological section will be completed with a discussion of the accuracy potential of the presented method (Sect. 2.5). In Sect. 3 we discuss the suitability and possible areas of the application of the method for the examples on the basis of different glaciology research pilot studies that were conducted applying the presented time-lapse measurement method and image sequence analysis approach.

\section{Methodological approach}

\subsection{Monoscopic image sequence approach - basic concept}

In the monoscopic time-lapse measurements, a single firmly installed camera observes the area of interest and continuously records images at a preselected time interval. The obtained image sequence is the basis for the determination of glacier motion. By applying appropriate feature tracking algorithms, the position of a large number of moving glacier surface points can be obtained for each image of the sequence. Thus, the glacier point's motion vectors in image space can be derived for each individual pair of sequence images. A trajectory of a glacier surface feature is obtained by tracking points through multiple images of the sequence.

The motion vectors or trajectories measured in image space have to be transferred into object space. This means they need to be scaled, and the 3-D position of the trajectory origins need to be determined. In a stereo-approach, a 3-D position is determined for each homologous point pair detected in the stereo-images (Fig. 1). This 3-D position also delivers the distance of the point to the cameras, which is required for scaling the measurements from pixels to metric values. However, stereo-image measurements on rugged glacier surfaces will often suffer from significant de-correlation. This means strongly different appearances of glacier surface features in the stereo-images affect the 
(a)

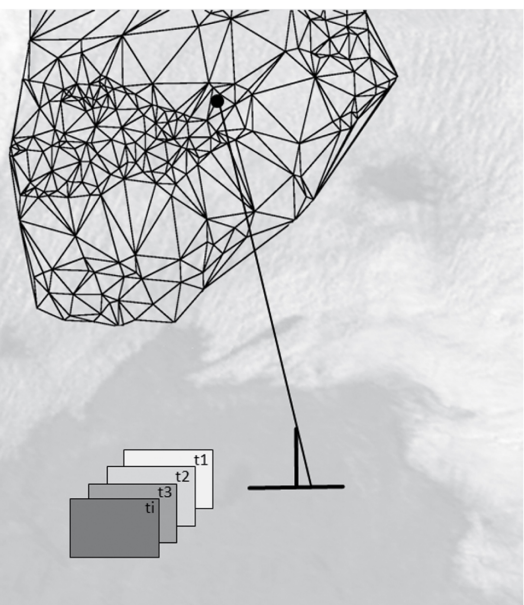

(b)

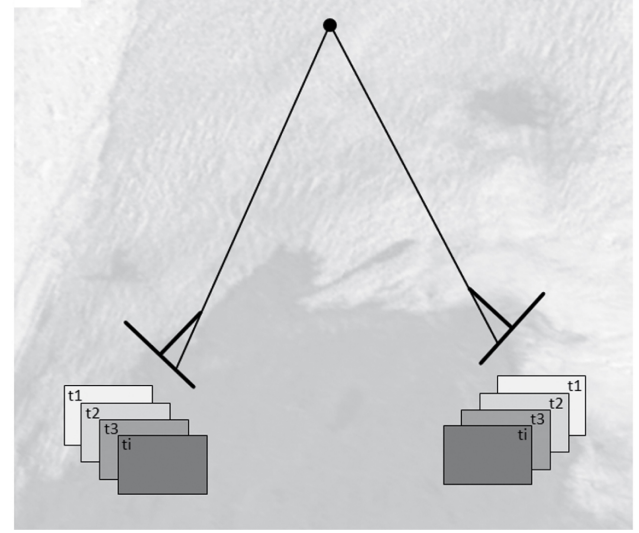

Figure 1. Monoscopic (a) vs. stereoscopic (b) image sequence analysis.

automatic detection of corresponding points, thus limiting the precision and reliability of stereo-image sequence processing. Therefore, monoscopic image sequence processing is more appropriate for phenomena which are mainly twodimensional due to the lack of significant transverse components in typical glacier motion patterns. Nevertheless, an approximate digital surface model (DSM) of the glacier as well as the camera position and orientation parameters are required for scaling and geo-referencing of monoscopic image sequence measurements. Based on this information, an image ray can be reconstructed for each pixel and intersected with the DSM (Fig. 1). This delivers the distance between glacier and camera for the scaling as well as the 3-D glacier point position for the geo-referencing of the measurement value. With knowledge on the time interval between consecutive images, metric velocity values can be calculated from the motion vectors or trajectories.

We limit ourselves to monoscopic image motion capture and to processing delivering two-dimensional velocity field information here, as the glaciology phenomena observed in the practical experiments do not show significant acrosstrack motion and can thus be well described in 2-D (compare Sect. 2.4.3). However, the method can be extended to 3-D trajectory measurements from stereoscopic image sequences straightforwardly by inserting an additional stereoimage matching step into the data processing chain.

Figure 2 shows the general workflow of the proposed concept for monoscopic time-lapse measurement and processing that will be described in detail in the following sections. The approach consists of two main tasks: the first one comprises the acquisition of the image sequences and their processing (also referred to as "measurements in image space"). The second one comprises the acquisition of geo-referencing data and their use for scaling and geo-referencing of the imagebased measurements (also referred to as "transformation into object space").
The algorithms regarding image sequence processing, scaling and geo-referencing presented here have been implemented in $\mathrm{C}++$. For the bundle block adjustment we used a photogrammetric in-house bundle library (Schneider, 2008). In Sect. 2.4.1 we will also discuss how the processing steps for the geo-referencing can be solved using commercial structure-from-motion (SFM) software (e.g. Agisoft PhotoScan).

\subsection{Data acquisition concept}

In order to guarantee a high accuracy of the final trajectories, we propose an integrated geo-referencing and timelapse measurement approach. Thus, the measurement setups for the time-lapse measurement and the geo-referencing cannot be considered independently of each other. Figure 3 shows the basic combined time-lapse and geo-referencing measurement set-up (Fig. 3a) and the required visual content of the measurement image acquired by the time-lapse camera (Fig. 3b) to meet the demands of successful precise data processing.

The main requirement for a camera to be used for glacier motion analysis is the ability to autonomously record images at defined time intervals over a certain period of time. It should therefore be equipped with a programmable timer as well as a weatherproof housing, a sufficient power supply (e.g. a batteries supported by a solar panel) and sufficient data storage capacity.

For a flexible and stable installation of time-lapse cameras, tripods thoroughly covered with stones have proven to be suitable. To determine both horizontal and vertical components of glacier motion and to reduce occlusions on the glacier surface, an elevated camera position is required, which allows for an oblique viewing angle on the glacier surface. Despite the stable installation of a time-lapse camera, slight camera motion (mainly induced by wind and temperature effects) cannot be completely prevented. To compensate 


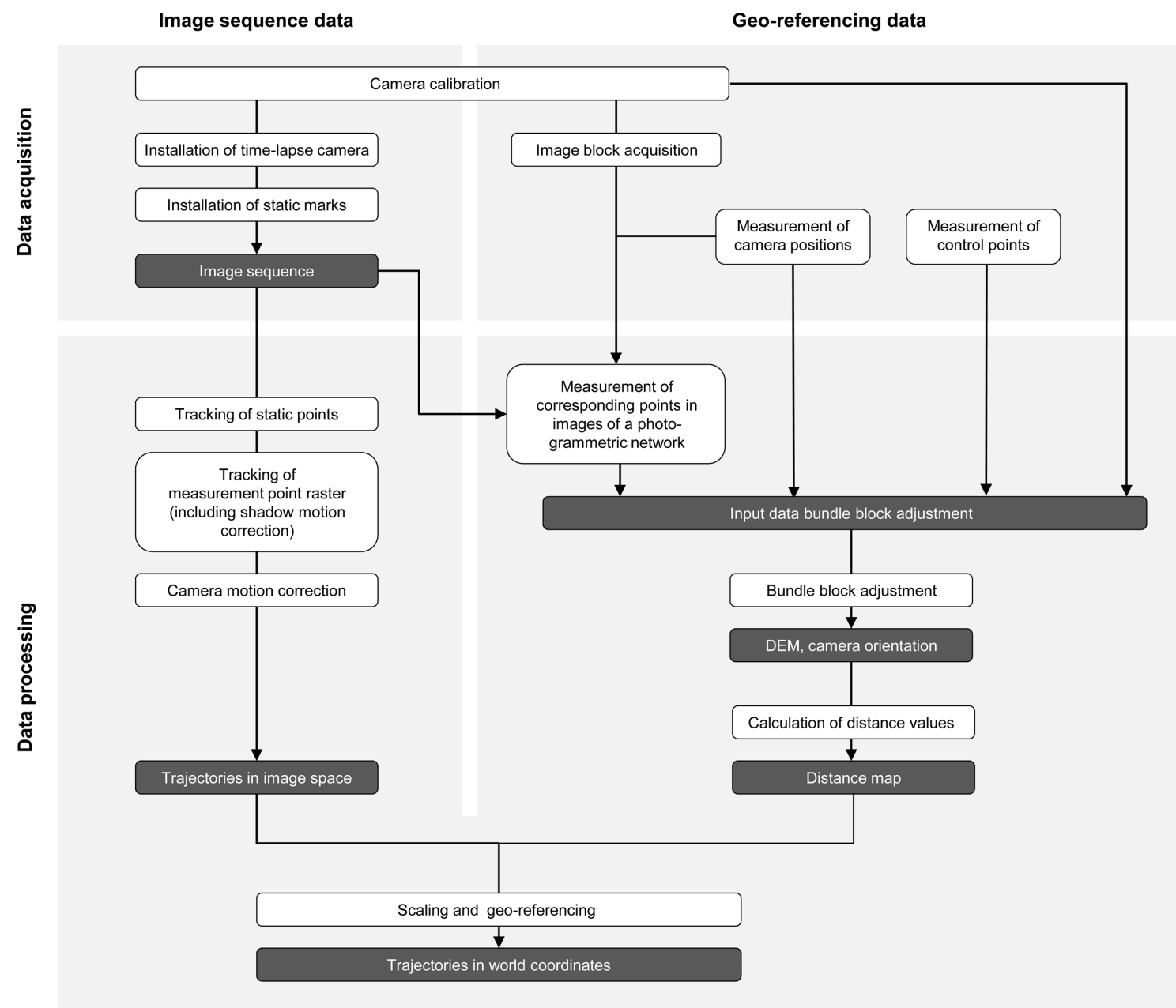

Figure 2. Workflow of monoscopic image sequence measurement.
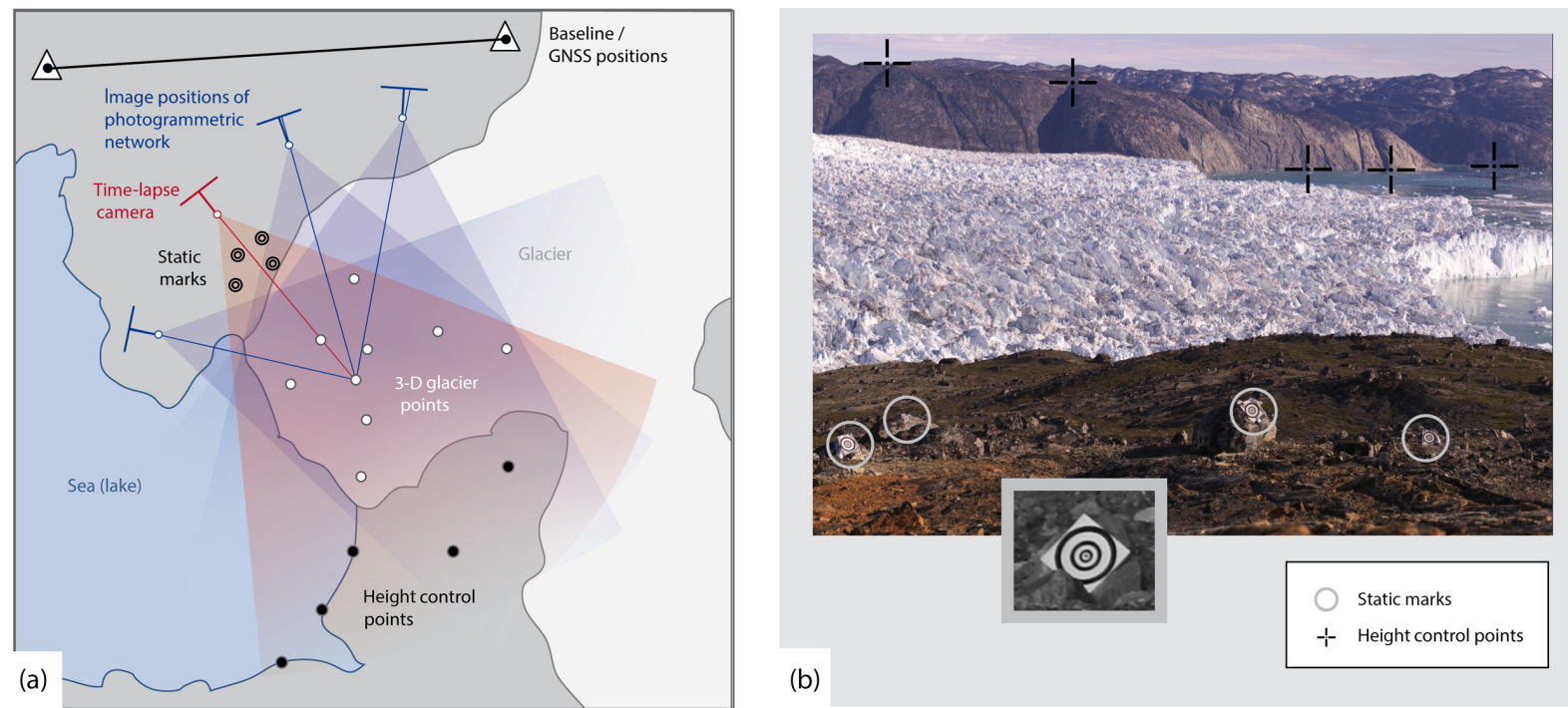

Figure 3. Combined scheme for the set-up of a time-lapse camera and its corresponding geo-referencing measurement set-up (a); measurement image of time-lapse camera (b). 
for this, it is necessary to have static points visible in the images, which can be used for correcting the influence of camera movement (see Sect. 2.3.3). These may be natural features in the image fore- and/or background, but in most cases artificial marks placed in the foreground (Fig. 3b) turned out to be more accurate and reliable.

For the geo-referencing and scaling of measurement values of the monoscopic time-lapse camera approach, the geometric relation between image and glacier surface must be known. Thus, a digital surface model of the glacier and the orientation parameters of the time-lapse camera have to be determined. This can be achieved by establishing a local photogrammetric network (consisting of several convergent images taken from different positions as shown in Fig. 3) plus some additional measurements. The acquisition of the corresponding multi-view images is conducted shortly after starting the time-lapse camera. Together with one temporarily fitting image of the recorded time-lapse sequence, these images form a photogrammetric network, which allows for the simultaneous determination of glacier surface point coordinates and camera orientation parameters by photogrammetric bundle adjustment (see, e.g., Kraus, 2007 or Luhmann et al., 2006). A minimum constellation to transform the results into a scaled and horizontal world coordinate system (i.e. to establish a geodetic datum) is given by the knowledge of two camera positions (preferably those forming the longest baseline) and one height control point (preferably at a far distance). However, it is recommendable to use redundant information in the process of geo-referencing. Thus, depending on the local topography, different variants of measurement set-ups are possible which require different measurement equipment and effort.

Variant 1: A minimal geo-referencing measurement equipment consists of a handheld GNSS (global navigation satellite system) device and a handheld laser distance measuring device. This instrumentation is sufficient if a highly accurate elevation reference is not required for a certain measurement task. Using the handheld GNSS, at least two camera positions (preferably those, which form the longest baseline) and at least one control point (ideally 5-10 for redundancy) in the background of the measurement object need to be measured. With the handheld laser distance measuring device precise distances between camera positions can be determined. The distance between the camera positions (called base length) defines the scale for translating image measurements into object space, and errors in the base length will propagate linearly into the length of determined motion vectors and trajectories.

Variant 2: A special, advantageous case occurs when the shoreline of a lake or the sea is visible in the time-lapse images. The control point measurements can then be reduced to a single water level measurement that delivers the $Z$ coordinate for several height control points along the shore line. These height control points form a good basis for defining the world coordinate system horizon.
Variant 3: If a higher positioning accuracy is required, the camera position determination via handheld GNSS measurements should be replaced by differential GNSS measurements. Besides an accurate elevation reference, camera positions measured with an accuracy of a few centimetres ensure a more accurate scaling of the photogrammetric network, thus making additional laser distance scale measurements dispensable. Depending on the camera's resolution, it might still be sufficient to measure control points using handheld GNSS if they are located at an adequate distance from the time-lapse camera.

Variant 4: The highest measurement effort is required for measuring environments in which the control points are inaccessible or very distant. In these cases control points need to be determined via triangulation. For this purpose a baseline has to be established whose coordinates are measured via GNSS. From the baseline positions, angle measurements are conducted by a tachymeter instrument to determine coordinates of control points via spatial intersection. Camera positions can then be transformed from a local horizontal coordinate system into a global coordinate system by the GNSS measurements.

\subsection{Image sequence analysis}

This section will focus on the measurements in image space. The basis is a recorded image sequence of a moving glacier. To derive motion vectors from an image sequence, an appropriate feature tracking algorithm is required as well as an appropriate tracking strategy. To obtain reliable results, suitable methods need to be developed to compensate for the main error influences, including the effects of camera motion as well as effects of the motion of shadows on the glacier.

\subsubsection{Feature tracking}

A wide range of algorithms are available for point tracking in image sequences (e.g. Brown, 1992; Zitová and Flusser, 2003). Generally, they can be separated into feature-based and area-based methods. Area-based methods establishing correspondences between small image patches by minimizing some cost functions are able to provide results with subpixel accuracy and allow for a dense and regular distribution of measurement points over the image area of interest. Feature-based techniques extract discrete features from the image and track them by comparing values of a defined feature vector. We chose an area-based method for the task of glacier motion determination due to the potential for higher accuracy. The chosen method depicts a combination of crosscorrelation (e.g. Lewis, 1995) and least squares matching (LSM; Förstner, 1982; Ackermann, 1984; Grün, 1985). The cross-correlation method is a fast option to obtain approximate values that are required for LSM. The advantage of LSM is that it is an adaptive, robust and accurate approach. It directly estimates subpixel-accuracy patch translation pa- 
rameters and simultaneously delivers figures on their accuracy and reliability. Furthermore, LSM is also capable of considering rotations and linear distortions of patches.

In order to determine glacier motion fields, a dense raster of points needs to be defined in the first image of the image sequence. For each of these measurement points a patch has to be defined as well as a search area before applying the actual tracking algorithm. The patch contains the area of pixels around the measurement point to be tracked, and the search area defines the region where the corresponding point might be located in the subsequent image. There are special demands on both of these when monitoring glaciers.

The resulting motion vector for a certain patch will be an interpolated value for the area on the glacier surface that is covered by the patch. When terrestrially observing glaciers, the view of the glacier surface will usually be rather oblique. Thus, a quadratic patch that contains an adequate amount of pixels will cover an area on the glacier surface that has a large extension in depth direction (i.e. across-glacier motion). This is especially critical for remote measurement points. In order to address this problem, a specific rectangular shape is chosen for each patch (Fig. 4). To automatically define the optimal patch size, two parameters are predefined. The first one is a number of pixels a search patch should contain to ensure stable matching, and the second one is a maximal difference in depth a patch should cover to avoid interpolation over large areas. While the depth parameter restricts the extension of the patch in the $y$ direction, the given number of pixels lets the patch grow proportionately in the $x$ direction. The DSM of the glacier surface that is required to obtain the necessary distance information for patch shape restriction can be provided as described in Sect. 2.4.2.

For the purpose of glacier motion measurements, the search area can be strongly limited in most cases. It mainly depends on the chosen time interval of the image sequence. Usually, the temporal resolution of the image sequences is high enough to keep the glacier movements between subsequent images small. In most cases the motion direction is predictable and the velocity of a glacier surface point does not change rapidly between subsequent images. Furthermore, the search area also depends on the influence of the camera motion, which can be determined beforehand (see Sect. 2.3.3). This a priori knowledge can be used to restrict the search area in order to reduce mismatches and ambiguities during tracking.

Depending on the application, different temporal or spatial strategies can be applied for the tracking points in an image sequence. Temporal strategies can be distinguished depending on how images of a sequence are combined for tracking. For instance, a trajectory can be determined by always tracking a point from the first image of a sequence into each of the other sequence images, or it can be determined by successively tracking the point from each image into the subsequent one. The first version in comparison to the second one is stronger regarding error propagation but weaker regarding image de-correlation. Thus, the first strategy has to be applied when tracking signalized points - as for camera motion determination (see Sect. 2.3.3) - and the second one when tracking natural points like glacier surface points.

Spatial strategies mean that either a certain feature (e.g. a specific crevasse) can be tracked through the image sequence (Lagrangian approach) or that the tracking is performed at a fixed position for each image of the sequence (Eulerian approach). The advantage of the first method is that it provides good visual control over the success of the tracking. The advantage of the second method is that it is independent on the loss of features (e.g. because of calving). This is especially important for long-term observations. However, for most applications, motion values are required that refer to a fixed position in space, which makes the Eulerian approach the standard for the tracking of glacier surfaces. For a subset of the measurement image, Fig. 5 shows trajectories resulting from feature tracking on a fixed position. The single measured translations of a trajectory, which are depicted here arranged one after the other, all refer to the starting point position of the trajectory.

\subsubsection{Handling of shadow motion effects}

The changing positions of the sun during the day as well as moving clouds generate moving shadows on object surfaces. In the case of environmental monitoring time-lapse observation, the motion of shadows may be a rather important issue. It influences the tracking method described above in such a way that the obtained motion vectors will be a combination of actual glacier motion and shadow motion. More specifically, it is not the whole area covered by a shadow that causes matching errors but the image areas that change from shadow to non-shadow and vice versa (see Fig. 6). Thus, to obtain pure glacier motion, the pixels of these areas need to be detected and excluded from the matching (see Fig. 6b).

The method developed to exclude shadow-influenced pixels during the matching will be described with the aid of a synthetic image pair as shown in Fig. 7, wherein the slave image is a copy of the master image but is shifted in a predefined way (glacier motion simulation). Additionally, both images are overlaid by a transparent black square whose position in the slave image is shifted by a certain amount in comparison to its position in the master image (shadow motion simulation). In this way a shadow overlaid glacier motion is simulated where reference values for both motion components are available.

In order to determine shadow motion pixel, the following iterative approach is applied: initially all pixels of a patch will be incorporated into the matching process via LSM. The corresponding patch at the thus determined position in the slave image is still completely influenced by the shadow motion. In the ideal case the grey value differences between original and corresponding patch would be zero after LSM transformation. In the first iteration step this perfect match cannot 

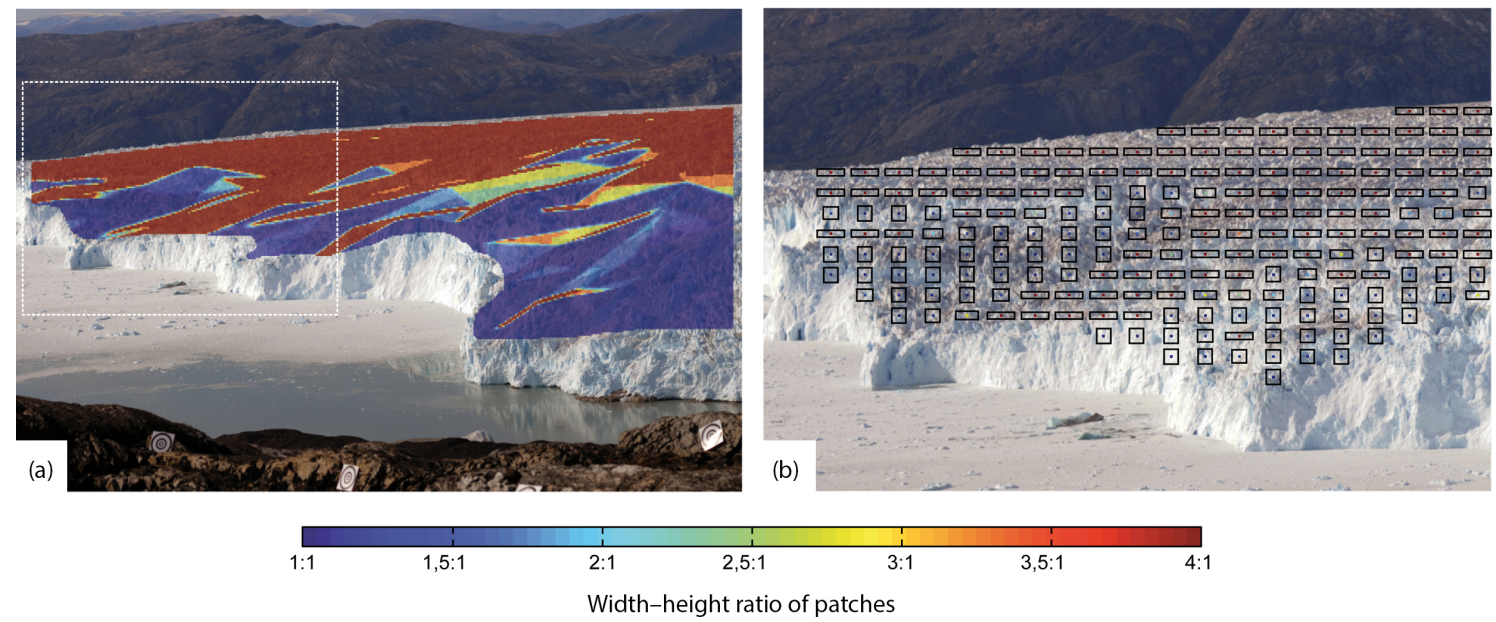

Figure 4. Locally adaptive patch sizes. The figure shows the result of the automatic patch size definition taking into account distance differences in the patch. (a) The determined aspect ratios for a patch are shown colour-coded at the corresponding position in the image. For a smaller image excerpt (white rectangle), the resulting patches (black contour) are superimposed on the sequence image (b).
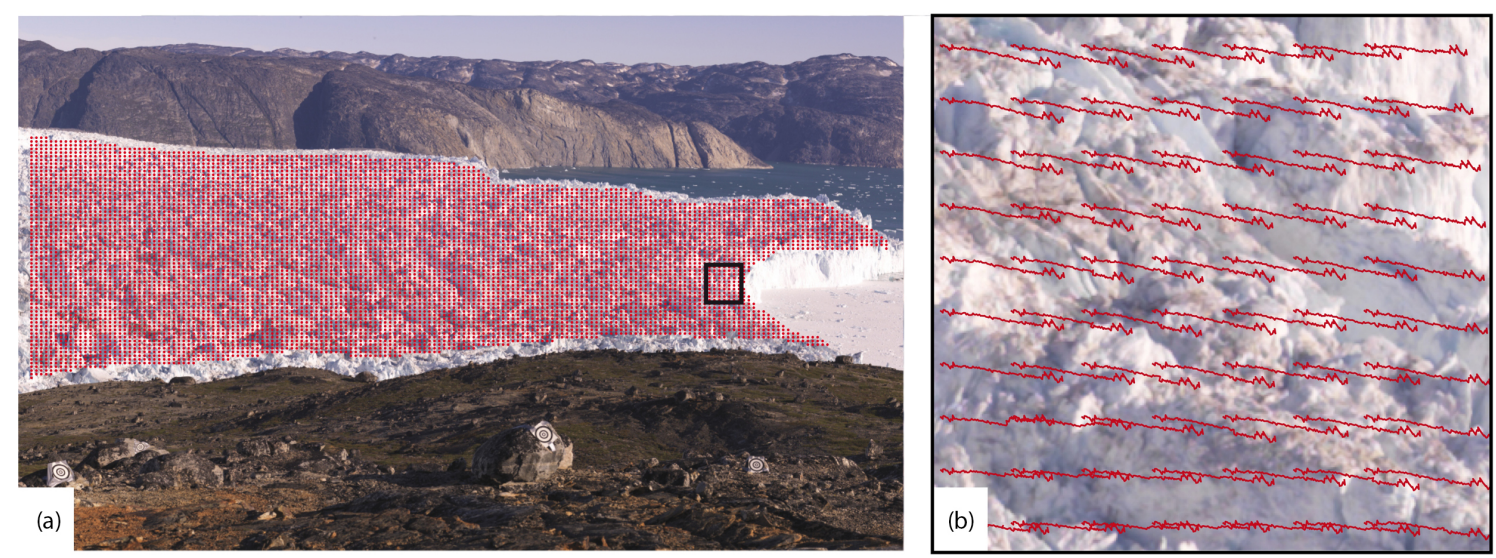

Figure 5. Raster of measurement points on the glacier surface (a) and resulting trajectories obtained from feature tracking (b). The figure shows the grid of the measuring points (red dots) as a superposition of a sequence image (a). For the area marked by the black square, the corresponding image section is shown on the right. It is superimposed by the measured trajectories (red lines) for a $24 \mathrm{~h}$ image sequence (acquisition interval $20 \mathrm{~min} ; \mathbf{b}$ ).

be achieved because the influence of moving shadows leads to an erroneous position of the corresponding patch. Thus, high grey values occur in the difference image of the master patch defined in image 1 and its corresponding patch detected in image 2, especially at the margins of the shadow areas (Fig. 7; first iteration). This information can be used to detect and exclude shadow-influenced pixels to guarantee a better solution in the next iteration step. Since highdifference values do not solely cover shadow shift areas but also some important glacier texture, we have to find an appropriate threshold that removes disturbing shadow-influenced pixels but keeps enough good glacier texture for the matching as well. When looking at the histogram of the difference image, the difference values are approximately normally distributed in most cases. The shadow-influenced pixels show the highest grey value differences and accumulate at the upper and lower margin of the histogram. Thus, using the single standard deviation of the difference values as a threshold ensures that no more than ca. $30 \%$ of the pixels of a patch are excluded and enough texture remains in the patch. Pixels with grey value differences higher or lower than the single standard deviation are defined as potential shadow motion pixels and are excluded from the matching procedure during the second iteration step. In this way the recalculated position of the corresponding patch is drawn to its real position. After the first iteration, some real shadow motion pixel but also a noticeable amount of non-shadow pixel is excluded. This ratio improves during the next iteration step along with the improvement of the matching result. The process continues until either the matching result does not change anymore or 

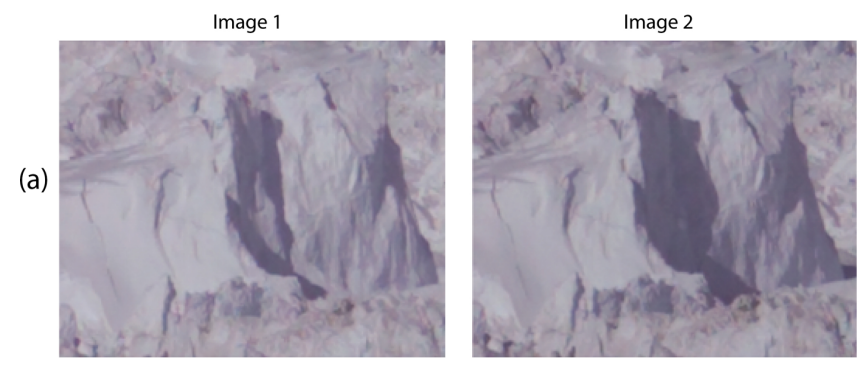

(b)
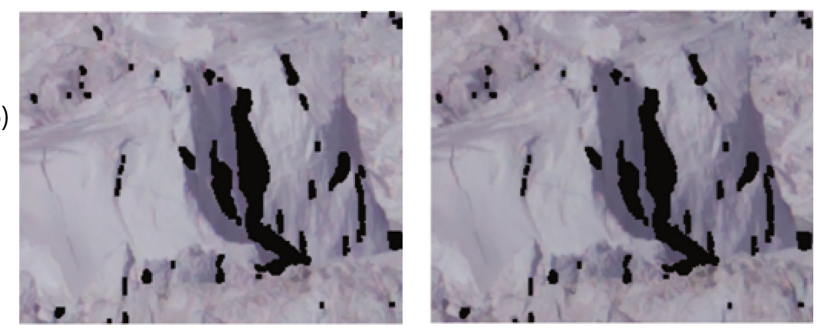

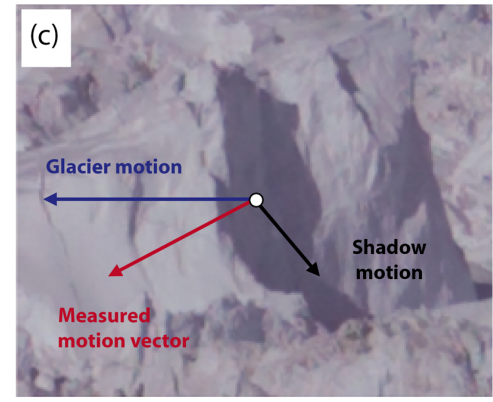

Figure 6. Influence of moving shadows in image sequences. The figure shows corresponding image sections from two sequence images recorded at a time interval of $30 \mathrm{~min}$ (a). In the same image pair those pixels were marked black which are influenced by shadow motion (b). These pixels falsify the result of the feature tracking because the tracked glacier movement is overlaid by the shadow movement (c).

no more new shadow motion pixels can be found (for the example shown in Fig. 7 this was achieved after the third iteration). The comparison of the reference values of the synthetic example in Fig. 7 with the tracked glacier motion values after the last iteration step show that the influence of the simulated shadow motion could be completely eliminated.

Of course, image sequences of natural scenes are influenced by illumination differences as well as by random noise of the sensor. This will also affect the quality of the matching result. Nevertheless the results can be significantly improved applying the method described above (Fig. 8).

\subsubsection{Camera motion}

The second main error source that might impact the motion vectors or trajectories is the effect of the motion of the timelapse camera itself. Even if a camera system is firmly installed, small movements caused by wind and temperature changes cannot be avoided. Thus, their impact on the measurements needs to be determined and corrected. As techniques such as LSM are capable of measuring image motion with a precision $<0.1$ pixel, camera motion has to be corrected for at an equivalent level.

The effects of camera motion can be determined (and corrected) by tracking static points in the images. These can either be signalized targets or natural points (Fig. 9). To facilitate camera motion compensation, a sequence image must not only contain the dynamic measurement object (e.g. a moving glacier) but also some terrain in the foreground and/or background. While signalized points can usually only be installed in the foreground area and are limited in number, natural points may be defined all over static image areas. Although natural points basically provide a better dis- tribution for the modelling of camera motion, their disadvantage is often a lower contrast and their sensitivity to illumination changes, moving shadows and visibility limitations. This will often lead to a lower precision and reliability potential for tracking natural points as compared to signalized targets. However, using the high redundancy of natural points in combination with a robust estimation method such as RANSAC (random sample consensus; Fischler and Bolles, 1981), this can be compensated for to some extent. Thus, for applications where tracking intervals of $24 \mathrm{~h}$ (i.e. minimized influence of illumination and shadow variations) are sufficient, natural points might be preferred. For tracking tasks with a higher temporal resolution, signalized points are often recommendable.

The tracking of static points results in displacement vectors for each subsequent image pair representing the camera motion between the two consecutive image recording times. These vectors can be used to derive parameters that appropriately describe the influence of camera motion for each individual pixel of the image. In general, two options to model camera motion are possible: the first option is to mathematically describe the real physical motion of the camera in object space, defining position and orientation changes in the camera as model parameters. The second option is to mathematically describe the effects of the camera motion in image space by a planar transformation. Applying the first method provides a potentially higher accuracy and distancedependent effects can also be modelled, which is not possible when applying a planar image transformation. However, in most cases it is sufficient to describe the effects of camera motion in image space via the two image shift parameters and the rotation parameter of a 2-D rigid transformation. 
Synthetic image pair:

\begin{tabular}{|c|c|}
\hline \multicolumn{2}{|c|}{ Simulated glacier motion: } \\
\hline $\begin{array}{l}\Delta x_{G \_R e f} \\
\Delta y_{G \_R e f}\end{array}$ & $\begin{array}{l}=-5,00 \text { pixel } \\
=\quad-2,00 \text { pixel }\end{array}$ \\
\hline \multicolumn{2}{|c|}{ Simulated shadow motion } \\
\hline $\begin{array}{l}\Delta \mathrm{x}_{\mathrm{S}} \\
\Delta \mathrm{y}_{\mathrm{S}}\end{array}$ & $\begin{array}{l}=6,00 \text { pixel } \\
=-10,00 \text { pixel }\end{array}$ \\
\hline
\end{tabular}
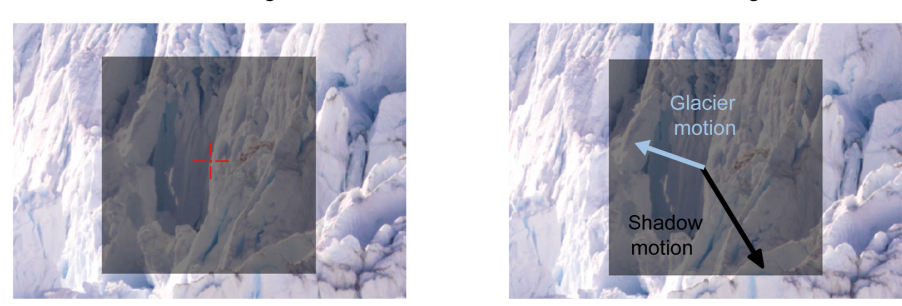

Measurement - LSM with shadow pixel exclusion:

\begin{abstract}
1. Iteration
(LSM without exclusion):

Measured glacier motion:

$\Delta \mathrm{x}_{\mathrm{G} \text { LSM }}=-0,98$ pixel

$\Delta y_{G_{-} L S M}=-9,52$ pixel
\end{abstract}

\begin{abstract}
2. Iteration:
(LSM with shadow pixel exclusion)

Measured glacier motion:

$\Delta \mathrm{x}_{\mathrm{G} L S M}=-4,06$ pixel

$\Delta y_{G_{-} L S M}=-5,02$ pixel
\end{abstract}

\begin{abstract}
3. Iteration:
(LSM with shadow pixel exclusion)

Measured glacier motion:

$\Delta \mathrm{x}_{\text {G_LSM }}=-5,00$ pixel

$\Delta \mathrm{y}_{\mathrm{G} \_ \text {LSM }}=-2,00$ pixel
\end{abstract}

Difference image
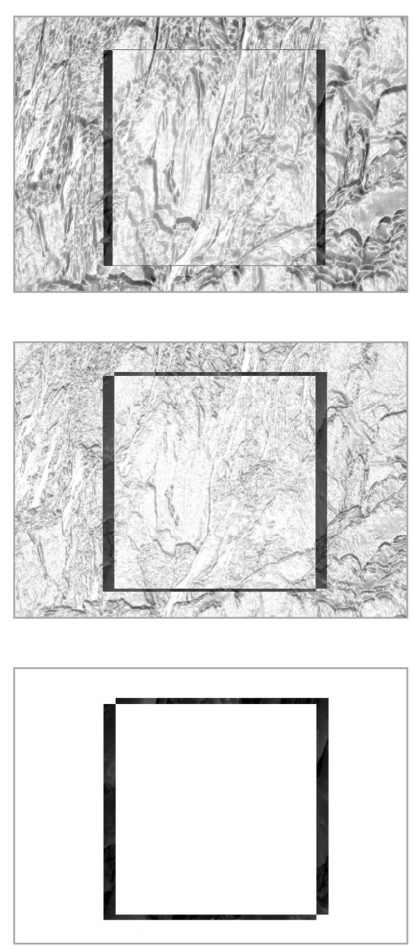

Excluded pixel
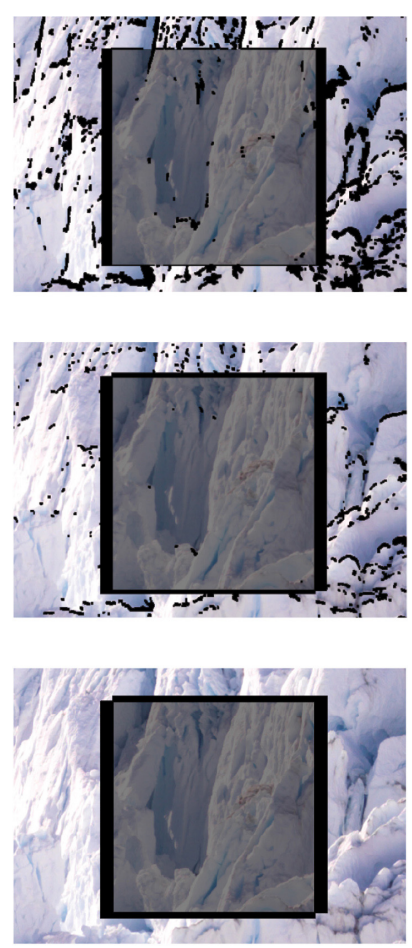

Figure 7. Least squares matching with shadow pixel exclusion. The figure shows a synthetically generated image pair, which simulates a glacier motion superimposed by shadow movement for which reference values are known (top). A method which iteratively detects and excludes shadow motion pixels from the matching is applied to this image pair (below). For each iteration step, the calculated translation parameters (left), the difference image between patch and transformed search patch (centre), as well as the resulting exclusion pixels for the next iteration, which are superimposed as black pixels on the patch (right), are shown.

In order to calculate the transformation parameters, the start and end coordinates of each tracked static point displacement vector are introduced to the equations of the affine transformation. Since each vector provides two equations, two static points would be sufficient to solve the equation system. Using natural static points, the rate of outliers among the tracked displacement vectors may be significantly higher than for signalized points. However, this can be compensated for by using a larger number of the natural points, eliminating outliers by robust techniques such as RANSAC. By applying these transformation parameters to the image coordinates of tracked glacier surface features, an individual correction value can be determined and applied to each of the measured motion vectors (Fig. 10). 


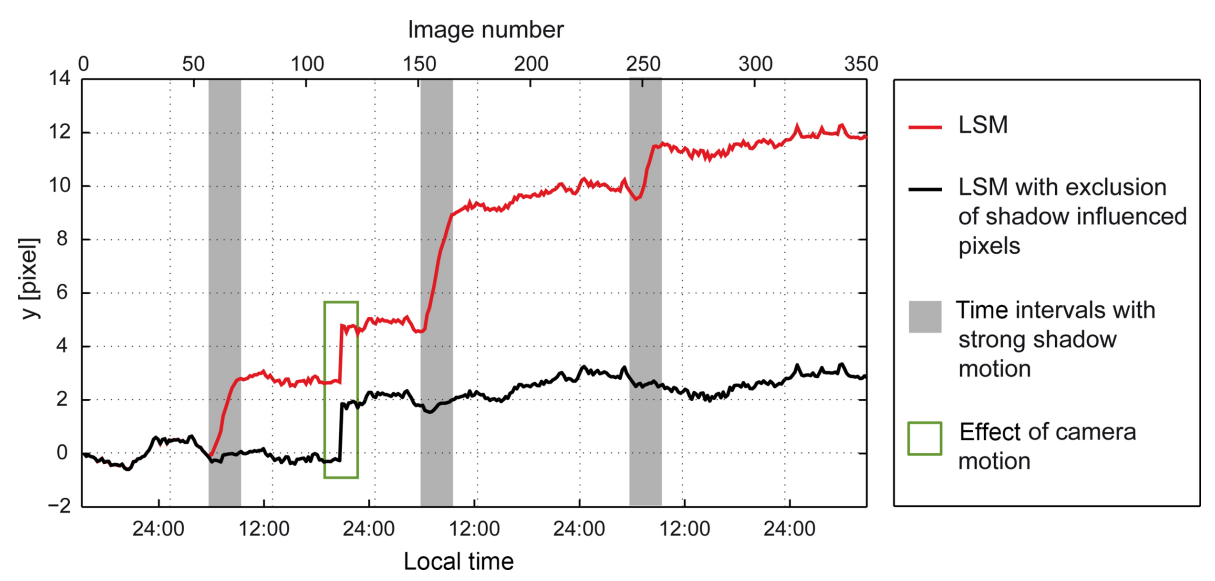

Figure 8. Drift effects during the measurement of a trajectory due to shadow movement. The figure shows the vertical component of a glacier trajectory measured using least squares matching (LSM) with and without shadow pixel exclusion. At certain times of the day (grey background), particularly strong shadows occur. Both motion curves still contain the influence of the camera motion.

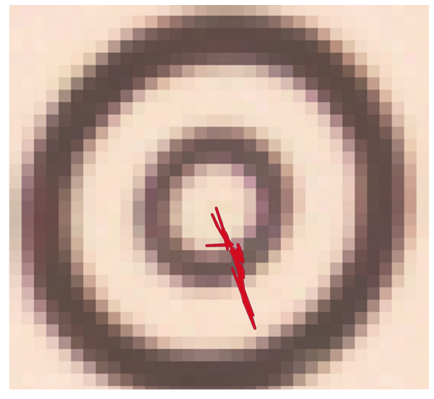

(a)

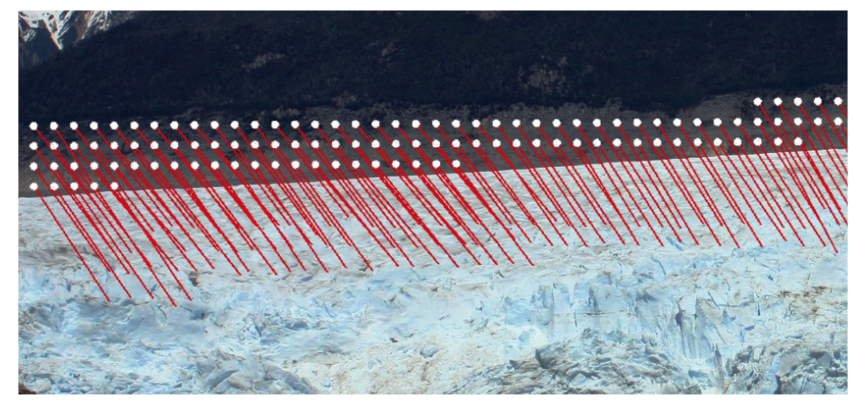

(b)

Figure 9. Tracking of static fiducial marks and/or static natural points. In panel (a), the figure shows an example of a signalized static point as it is visible in a sequence image. The red line here shows the motion path of the mark during a $24 \mathrm{~h}$ image sequence (acquisition interval $20 \mathrm{~min}$ ). In panel (b), an example of the use of natural static points is shown. The white dots represent a raster of static points that have been defined on the mountain slope in the background of the glacier. The red vectors (enlarged) represent the results for tracking these points from one sequence image into another one recorded $24 \mathrm{~h}$ later. The red motion lines of the static points (signalized or natural) correspond to the effect of camera motion.

\subsection{Geo-referencing}

After feature tracking, camera motion and shadow correction, glacier surface motion vectors and trajectories are available in image space. However, to allow for a reasonable interpretation of these motion vectors and trajectories, they need to be scaled from pixel to metric space (Fig. 11a) and linked to their absolute 3-D position in object space (Fig. 11b). Obviously, each vector or trajectory has its own scale factor due to their varying distance to the camera. While proper scaling is required for motion analysis, absolute geo-referencing is a precondition to fuse measurements with other data (e.g. remote-sensing image overlay) or to compare measurements of different epochs or measurement techniques. This section describes how the geometric reference between image and object space can be realized.

\subsubsection{Camera orientation and 3-D elevation model of the measurement object}

The basis for all further evaluation steps is the determination of object coordinates on the glacier surface. These are used to calculate a coarse digital surface model for the glacier area visible in the image sequence, which forms the basis for the determination of the distance between camera and glacier surface points as needed for scaling. For this purpose, further knowledge on the position, orientation and intrinsic calibration of the time-lapse camera is required. In order to determine these parameters, we make use of a photogrammetric network set-up as described in Sect. 2.2.

The recorded multi-view images, including an image from the time-lapse camera, are processed via photogrammetric bundle block adjustment (e. g. Kraus, 2007), allowing for simultaneous determination of camera orientation parameters and glacier surface point coordinates at a high accuracy. In 
(a)

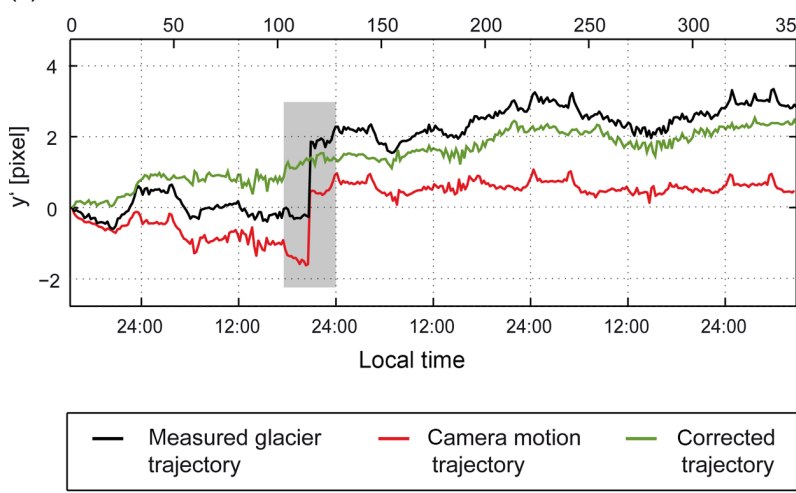

(b)

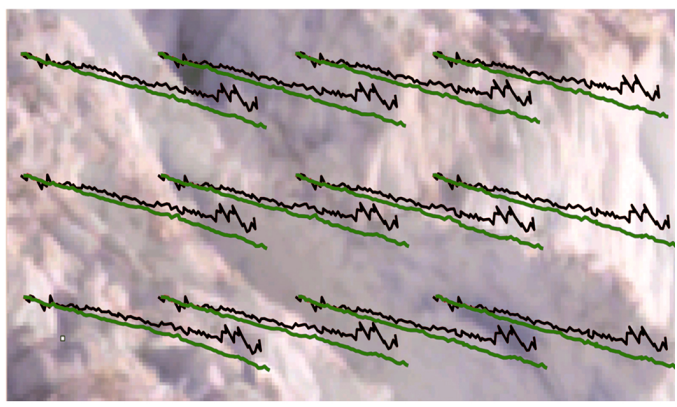

Figure 10. Application of camera movement compensation. (a) Shows the vertical component of a measured trajectory (black), the camera motion trajectory (red) and the corrected trajectory (green). The grey area indicates where a jump in the camera movement occurred. (b) A small subset of a sequence image is shown overlaid by some measured trajectories (tracked through a $24 \mathrm{~h}$ image sequence). The originally measured trajectories (black) clearly show fluctuations caused by camera motion. In contrast, the corrected trajectories (green) finally represent the smooth glacier movement.
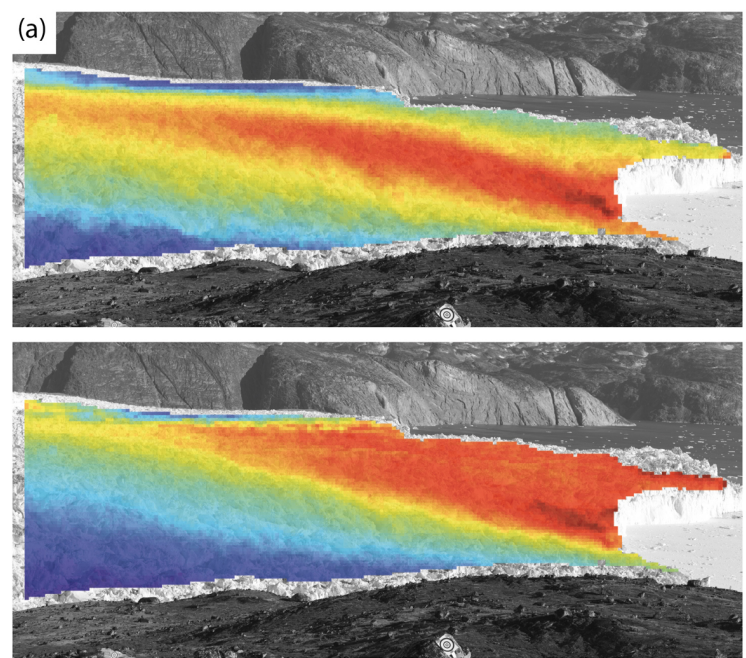

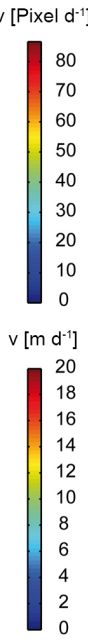

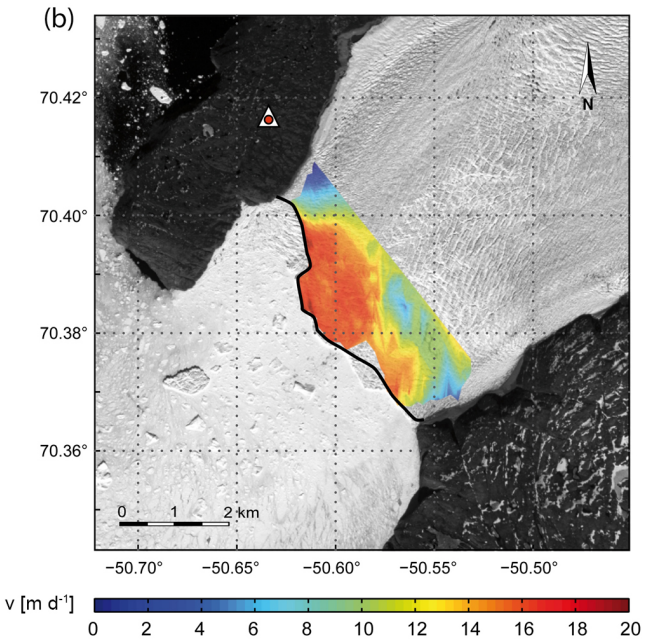

Figure 11. Result of scaling (a) and geo-referencing (b) at Støre Qarajaq glacier. The figure shows the colour-coded superposition of velocity values derived from measured trajectories. They are shown as unscaled values in image space (a, top) and scaled values transferred into object space (a, bottom), as well as geo-referenced values that can be overlaid on satellite images (b).

contrast to other approaches (e.g. Ahn and Box, 2010, or Messerli and Grinsted, 2015), which use an external DSM that needs to be registered with the time-lapse image, we propose an integrated solution to derive the DSM and its registration with the time-lapse image in one step. This offers high inner accuracy and avoids registration errors. The thus obtained glacier surface elevation model is optimally adapted to the time-lapse camera field of view and its oblique viewing angle.

For the processing we originally solely used a photogrammetric in-house bundle library (Schneider, 2008). With the advancing development of structure-from-motion tools we also started to integrate some of the SFM functionalities into our workflow. For this purpose we now use a combi- nation of Agisoft PhotoScan and the photogrammetric inhouse bundle. For example, we took advantage of PhotoScan to automatically measure image coordinates of corresponding points in the images of the image block, which were exported into the bundle block adjustment. Going a step further and calculating the sparse cloud while providing the measured camera positions to PhotoScan, the thus determined 3D coordinates of object points can also be exported and used as approximate values for the bundle block adjustment. The major advantage of an open photogrammetric bundle is in the fact that it allows for a thorough error analysis and flexibility regarding different measurement configurations. It can be adapted to different types of control points as well as different sets of camera calibration parameters, scale conditions 
can be defined, and it provides the possibility to define each variable as fixed or parameter to be estimated. Since many SFM tools are optimized for fast processing and 3-D visualization rather than for accurate measurement purposes, some limitations may have to be taken into account when applying them for measurement tasks (compare, e.g., Eltner and Schneider, 2015; James et al., 2017). However, when using a geo-referencing set-up that allows for the measurement of 3-D-control points instead of height control points, it is also possible to determine object coordinates and camera orientation parameters solely using PhotoScan. Preconditions for this are, a network configuration of at least three images and three to five 3-D control points. This can be achieved when applying the geo-referencing variants 3 and 4 as introduced in Sect. 2.2.

Due to restrictions posed by the terrain, the intersection geometry of the photogrammetric network established in the field is often rather poor. Thus, it is recommendable to measure the cameras positions in the field and to pre-calibrate the cameras in the lab or in the field. For camera calibration we used the photogrammetric software Aicon 3-D Studio and coded targets that can be measured with subpixel accuracy. To ensure continued validity of the camera parameters and to avoid changes in the interior orientation due to transport, it may be beneficial to install a calibration area in the field and calibrate the cameras shortly before taking measurement images or installing them as time-lapse cameras. The camera position coordinates and the parameters of interior orientation and lens distortion are then introduced as fixed values into the bundle block adjustment to stabilize the network and to minimize correlations between parameters. Seven degrees of freedom need to be fixed for the network (three translations, three rotations, one scale). This can be achieved with at least two 3-D control points and one height control point. Thus, the minimum set-up which can be used consists of an image network of two images with known positions and a single height control point. However, to ensure a successful and accurate processing of the photogrammetric network 510 control points that are well distributed within the field of view are recommendable.

As result of the photogrammetric bundle block adjustment, the orientation parameters of the time-lapse camera and 3-D coordinates of a large number of object points on the glacier surface plus an individual error value for each parameter and coordinate are obtained. If using only using PhotoScan, camera orientation parameters and very dense 3-D point clouds of the glacier surface are derived as well but without an integrated error analysis.

\subsubsection{Distance map}

In order to generate the DSM, the calculated 3-D object points are meshed to a TIN (triangular irregular network; e.g. Delaunay, 1934). Using the DSM the individual distance between the camera and the corresponding object point on the glacier surface is assigned to each pixel in the time-lapse image. For this purpose, the image ray for each pixel can be reconstructed based on knowledge about the exterior and interior orientation of the time-lapse camera and intersected with the DSM. From this intersection point and the camera projection centre, the distance value for the corresponding pixel is obtained (see Fig. 12). The distance image thus created forms the basis for scaling the individual trajectories and can also be used for automated parameterization of individual patch shapes in image sequence analysis (compare Sect. 2.3.1).

In the case of using solely using PhotoScan for bundle block adjustment and distance calculation, the results we need to obtain from the PhotoScan bundle are the exterior orientation parameter of the time-lapse camera, and from the dense matching step we need to obtain the depth map of the measurement object (a depth value for each pixel of the timelapse image). Both can be exported for the time-lapse image. After converting the depth map (containing depth coordinates of object points referring to the camera coordinate system) into a distance map (containing distance values between object points and the camera's perspective centre), the output can be further used as described in the following.

\subsubsection{Scaling and position determination}

In the case of a horizontally oriented camera and an orthogonal viewing direction of the camera to the flow direction of the glacier, the motion vectors can simply be scaled by the distance values. However, this can usually not be perfectly realized in the field and is often not desirable either, since the sequence camera's field of view should cover the measurement area optimally, which often necessitates an oblique viewing angle. When applying a simple scaling by distance, erroneous vectors (red vectors compared to the correctly scaled vectors in green) would be obtained as shown in Fig. 13a. The size of the error depends on the motion vectors position in the image and the deviation of the viewing angle from orthogonality to the glacier motion direction (Fig. 13b).

Thus, a more comprehensive method for the transformation of a vector or trajectory into object space is required. In the monoscopic approach, model assumptions have to be made about the direction of movement of object points. It is assumed that each glacier point moves in a vertical plane oriented along the flow direction of the glacier, i.e. that there is no significant motion across the moving direction (see Fig. 14). Starting from the first point $\mathrm{P}^{\prime}$ of a motion vector or trajectory given in an image coordinate system, the image ray is reconstructed and the corresponding 3-D object point $\mathrm{P}$ is determined by means of the known distance. The object point $\mathrm{P}$, the vector of the glacier flow direction and its perpendicular define the vertical movement plane for the measured point. The glacier flow direction can, for example, be obtained via flow-line patterns that are visible in satellite orthophotos. By intersecting the image ray of the second motion vector point or of each further trajectory point with the 


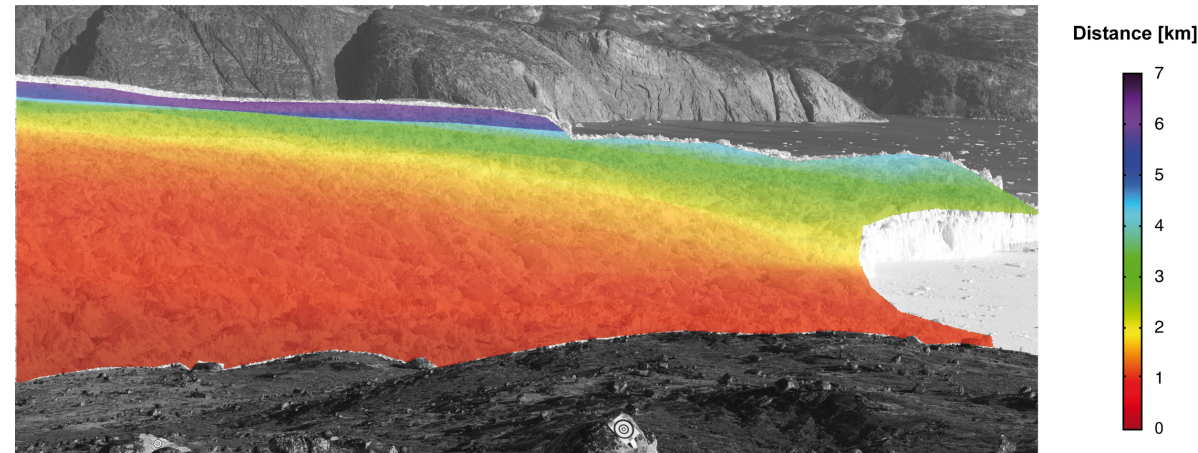

Figure 12. Distance map.
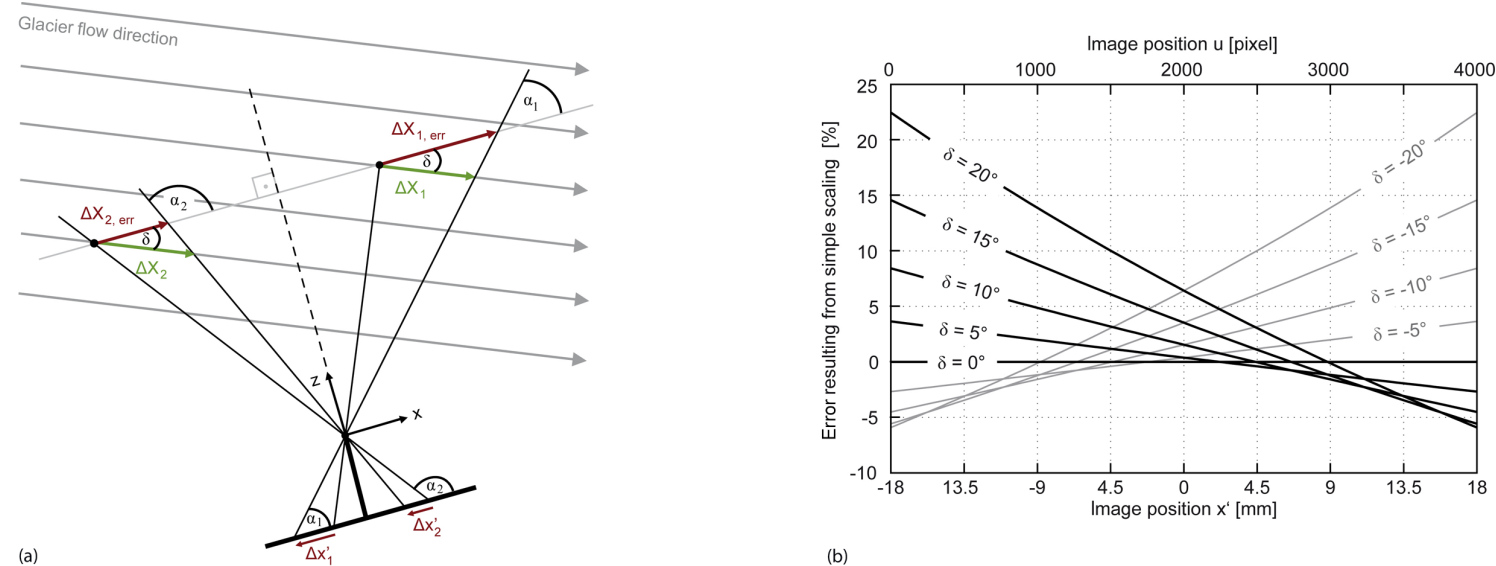

(b)

Figure 13. Scaling error in the case of a deviation $\delta$ from orthogonality between the glacier flow direction and the viewing direction of the camera (shown for the horizontal motion component).

thus defined vertical plane, the 3-D object coordinates can be determined for all points of a motion vector or trajectory.

\subsection{Aspects of accuracy}

The error in a determined motion vector is composed of several individual error influences. According to the individual steps of the image sequence analysis, four main error sources can be distinguished: the error in the image point tracking, the error in the camera movement correction, the scaling error and the error in geo-referencing. Whereas the first three errors mentioned have an influence on the accuracy of the determined translations, the geo-referencing error affects the accuracy of the spatial reference of the translation values. The errors in image point tracking and camera movement correction occur during the measurement in image space, the errors in scaling and the geo-referencing while transforming the measurements into object space. The effect of each of these errors on the measured translations is also influenced by parameters of the time-lapse measurement set-up, namely the principal distance and sensor size of the camera, the recording interval of the time-lapse sequence, the distance to the object, the local position of the measurement point in the image, and the amount of the measured translation itself.

Using the example of an image sequence measurement at Jakobshavn Isbræ in May 2010, we exemplarily want to show the magnitude of accuracy that can be achieved with the method. For this time-lapse measurement a Canon EOS1000D (pixel size $5.7 \mu \mathrm{m}$ ) was used equipped with a $35 \mathrm{~mm}$ lens which recorded images with an interval of $20 \mathrm{~min}$.

For a sample trajectory (at a distance of $3000 \mathrm{~m}$ from the camera) of this data set, the main error effects mentioned above have been estimated for each translation value of the trajectory applying statistical error estimation techniques (compare, e.g., Niemeier, 2002; Taylor, 1997).

For the determination of the error in image point tracking, the error analysis that is an integrated part of the LSM procedure provides accuracy values that result from the statistical evaluation of grey value differences between two patches. The standard deviation for a matching is thus a measure of the influence of the sensor noise and how accurately the transformation model of the LSM approximates reality. Al- 


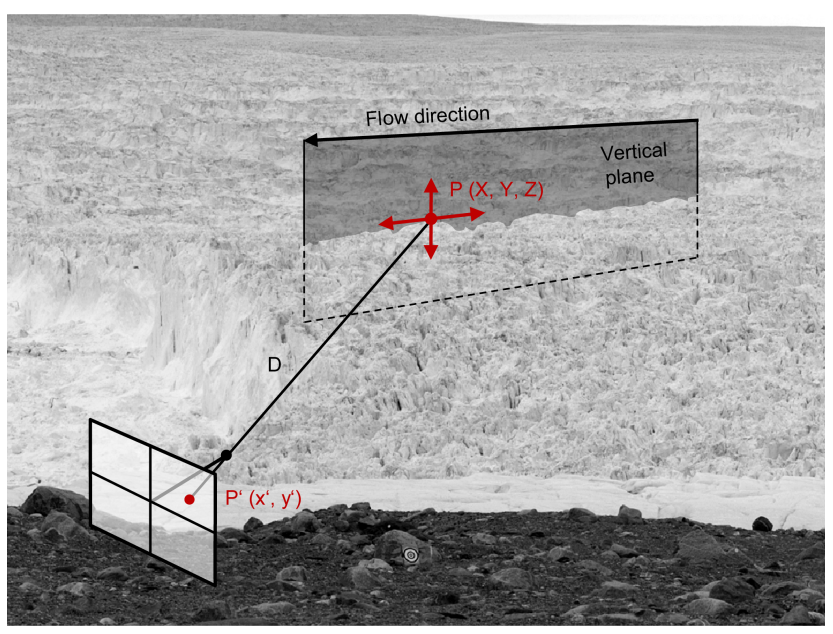

Figure 14. Transformation into object space.

though the influence of shadow motion is significantly reduced by the method explained in Sect. 2.3.2 a remaining error in shadow motion influence, and in the case of the vertical translation component also refraction effects, has to be considered additionally. Thus, on average, an error of 0.05 pixels for the horizontal and 0.17 pixels for the vertical motion component were estimated for the single translations of the trajectory. These errors in image space translate into error values of 2.3 and $8.1 \mathrm{~cm}$ in object space, in relation to the distance of $3000 \mathrm{~m}$ between measuring point and time-lapse camera.

The accuracy with which the camera motion correction can be applied is influenced by the tracking error in the static targets as well as by the quality of the functional model, which is used to mathematically describe the camera motion (see Sect. 2.3.3). On average, we estimated an error in camera motion correction of 0.14 pixels for the measured translations of the example trajectory. Regarding the distance of the measuring point from the camera, this corresponds to an error of $6.8 \mathrm{~cm}$ for each motion component.

The error in scaling is influenced by the scale error and the inner accuracy of the photogrammetric network as well as by the error in the angle that describes the deviation from the orthogonality of camera direction and glacier movement direction. The latter is strongly dependent on the position of the measuring point in the image (Sect. 2.4.3) and mostly influences the horizontal motion component. The scale error in the photogrammetric network depends on the accuracy of the measured camera or baseline positions and thus on the chosen geo-referencing measurement set-up (see Sect. 2.2). The inner accuracy of the photogrammetric network depends on the network configuration (convergent angles between image views and the number and distribution of image positions), the quality of camera calibration and the cameras sensor resolution. The total scaling error was estimated to be $7.27 \%$ for the horizontal and $0.34 \%$ for the vertical motion component.
For a measured motion component of $0.5 \mathrm{~m}$, this corresponds to a scaling error of $3.6 \mathrm{~cm}$ (horizontal) and $0.2 \mathrm{~cm}$ (vertical).

These individual errors were propagated into a mean total error of $9.2 \mathrm{~cm}$ for the horizontal translations of the example trajectory and $10.7 \mathrm{~cm}$ for the vertical translations. Note that the major error components are of absolute nature; this means that the relative error (i.e. error divided by velocity) will drop almost linearly with increasing velocity. It should also be noted that the camera movement and georeferencing-related error components show a local and temporal correlation; this means that spatial or temporal velocity gradients will have a higher precision, as correlated error effects will cancel out.

These error calculations exemplarily described above for a single trajectory were conducted for each trajectory of the measured area on the Jakobshavn glacier. Figure 15a shows the horizontal daily velocity values derived from the measured trajectories and the corresponding error values for the velocities calculated from the trajectories translation errors. Figure $15 \mathrm{~b}$ shows velocity errors of 5 to $15 \mathrm{~cm} \mathrm{~d}^{-1}$ and the decrease in the accuracy with increasing distance between measurement point and time-lapse camera.

A further issue to consider is the error in the georeferencing, which does not influence the measurement values themselves but is important when the measurements need to be compared with other data sets. This error strongly depends on the chosen method and instrumental set-up for the geo-referencing (compare Sect. 2.2). Generally, we need to distinguish between lateral errors and height errors. Thereby the lateral accuracy of the spatial reference points of the individual trajectories and motion vectors is influenced by the position errors in the baseline (formed by the positions of the cameras of the photogrammetric network or by the positions of the total station measurements). The error in a height control point causes a rotation error around the baseline and thus a height error in each measuring point. The error in a fardistant height control point thereby has a minor effect on the measurement points than the same error in a height control point with a smaller distance to the baseline.

\section{Discussion}

The velocity fields derived by monoscopic image sequence analysis can be further used for a wide range of motion analysis tasks. Depending on the application and characteristics of the measurement object, the basic method as described above may be individually adapted. In comparison to satellite-based analysis, terrestrial photogrammetric techniques cover only small areas but provide measurements at a very high spatiotemporal resolution and accuracy. Thus, the method is especially suitable for the investigation of objects which show a high motion dynamic. The derived motion vectors or trajectories contain information about horizontal as well as vertical motion components, allowing for manifold glaciologic 

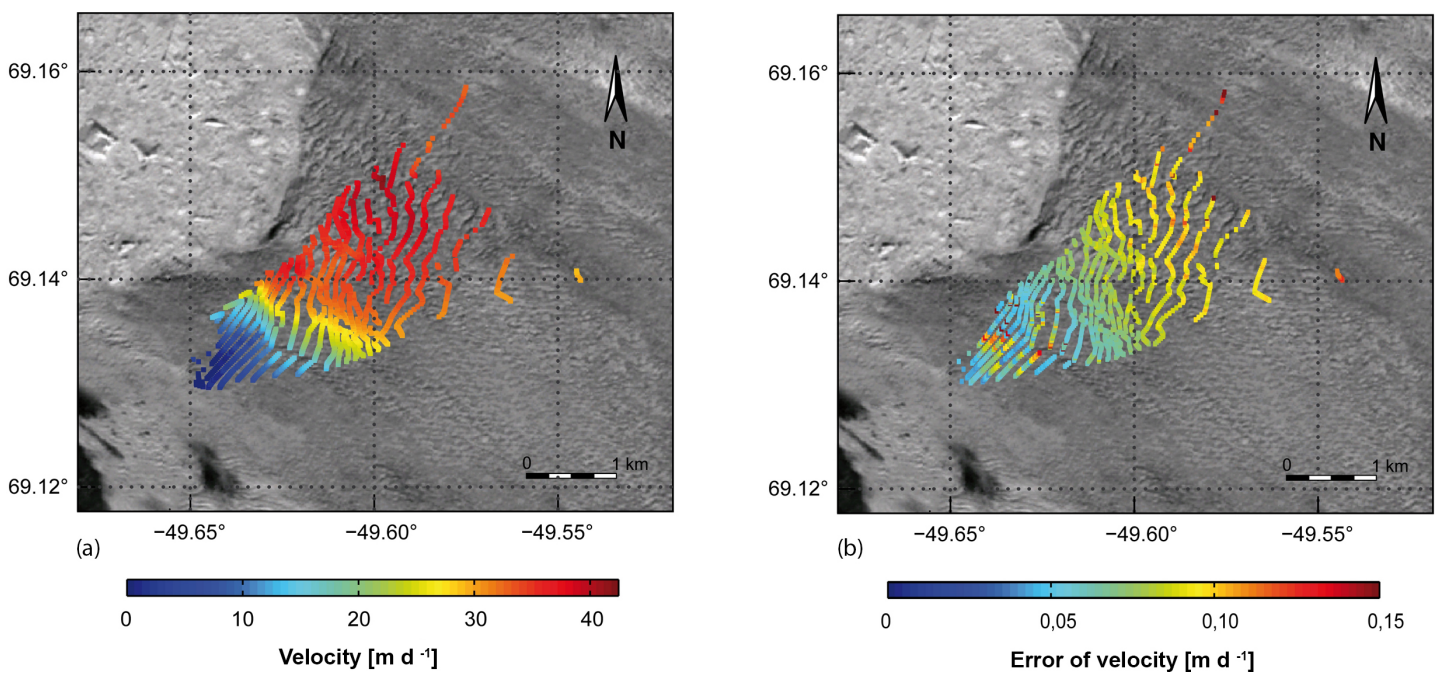

Figure 15. Horizontal velocity field (a) and corresponding error values (b) (example: Jakobshavn Isbræ, May 2010).

analyses. Since we are focusing on glaciology here, we want to discuss the potential of the method for different types of glaciology applications in this section. The discussion is based on the results of previous case studies on determining glacier motion data with the method presented here.

\subsection{Horizontal glacier motion}

Horizontal glacier motion fields derived from time-lapse imagery provide information about the motion of the glacier as well as spatial and temporal variations in velocity. They have proven to be a valuable database especially for the investigation of fast-flowing, highly dynamic glaciers, like many outlet glaciers of the big ice sheets.

Glacier motion velocity fields. If the recording interval of the time-lapse images is known, velocity fields can be derived directly from the glacier motion fields. Depending on the time interval of the time-lapse series, velocity fields can be generated with very high temporal resolution. They are especially suitable for very fast-moving glaciers such as many of the Greenland outlet glaciers that are of high scientific interest in the context of mass balance determination and sea level rise prediction. For these glaciers - with maximal velocities in the order of several tens of metres per day the method is a useful supplement to glacier flow velocities derived by remote-sensing methods which are hampered by their revisit time intervals. Figure 16a shows velocity fields close to the front of Jakobshavn Isbræ (West Greenland) obtained by monoscopic terrestrial image sequence analysis over 6 years, displaying glacier surface velocities of up to $40 \mathrm{~m} \mathrm{~d}^{-1}$. Each velocity field consists of up to 4000 velocity vectors. Similar velocities have also been determined by $3-$ $\mathrm{D}$ feature tracking in multi-temporal terrestrial laser scanner data (Schwalbe et al., 2008).
Another advantage of the high temporal resolution of the terrestrial image sequences is that this allows for filtering the data. For example, if daily velocities are required but the time-lapse camera is operated with a 10 to 20 min time interval, the redundancy in the measurement values can be used to detect and eliminate outliers or to improve the precision by averaging. Thus, investigations of glaciers with lower velocities may also benefit from the terrestrial time-lapse measurements. This may be especially important for regions with changing weather conditions and frequent rainfall or fog as it is, for instance, the case for Patagonian glaciers. As an example, Fig. 16b shows the velocity field of Grey Glacier (Southern Patagonian Ice Field), where each velocity value has been derived from about 300 single motion vector measurements (Schwalbe et al., 2017).

Analysis of calving events. Due to the (almost arbitrarily) short image (and thus measurement) intervals, the analysis of terrestrial image sequences also allows for the investigation of short-term changes in the velocity field during calving events. Calving events take place within a short period of time, thus requiring a high temporal resolution of the measurement method. On the other hand, the beginning of a calving event can hardly be predicted, which makes it necessary to autonomously record the glacier front area over a long period of time. An example of this kind of application is the case study presented in Rosenau et al. (2013), where velocity fields during a large calving event at Jakobshavn Isbræ (West Greenland) were analysed on the basis of an image sequence over several weeks in May/June 2010 recorded with a temporal resolution of $20 \mathrm{~min}$. During the calving event, the motion velocity close to the glacier front locally jumped up to $70 \mathrm{~m} \mathrm{~d}^{-1}$ within a period of a few hours. The velocity thus almost doubled in the area of the new glacier front. The impact of the calving on the motion behaviour of the glacier could still be determined up to $1 \mathrm{~km}$ upstream. 

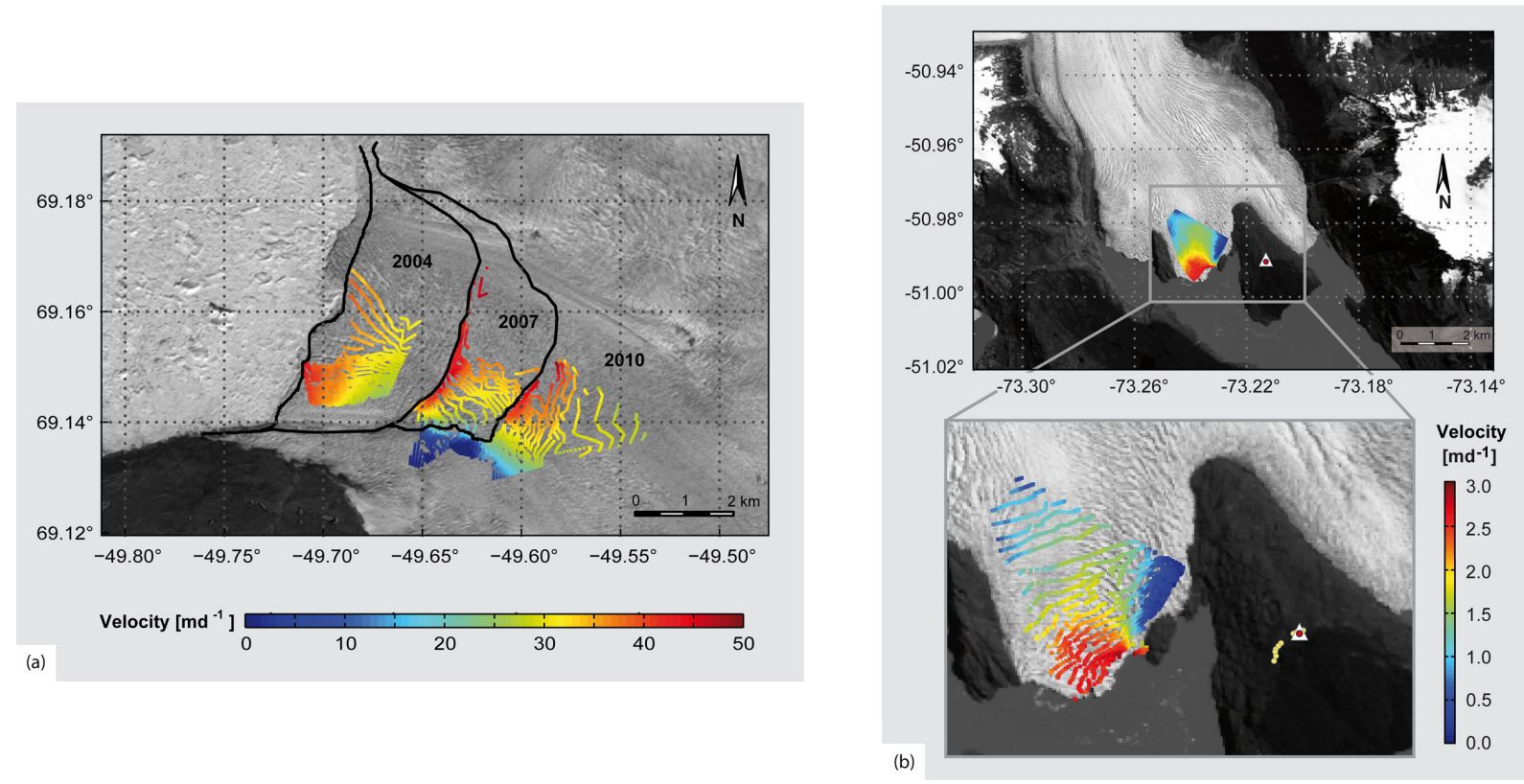

Figure 16. Velocity fields of Jakobshavn Isbræ, West Greenland (a) and Grey Glacier, Southern Patagonian Ice Field (b).

\subsection{Vertical glacier motion}

The vertical component of the glacier motion field can be especially useful to draw conclusions on events taking place underneath the glacier surface and to recognize specific features that lie within or under the glacier. The vertical movement field thus has the potential to give an indirect view into the glacier.

Grounding line determination. When investigating outlet glaciers that terminate into the sea, the vertical motion component of the trajectories may provide information about tidally induced movements of the frontal area of the glacier tongue. Using tidal models or sea level measurements as a reference, various parameters such as vertical tidal participation, phase shift and inclination can be estimated for the measured trajectories. These parameters characterize the differences between the movements of the glacier surface compared to the tidal curve. In particular, the scale factor in vertical direction, which can be interpreted as a damping factor of the amplitude, can be used as an indicator of the detection of floating glacier areas. The detailed procedure for calculating the percentage of the vertical glacier movement compared with the tidal range (free float) is explained in Dietrich et al. (2007). The transition from the free float section of the glacier (with almost $100 \%$ tidal movement participation) to the grounded section (with no tidal movement participation) can be used for the determination of the glacier's grounding line. In Rosenau et al. (2013) a case study is presented where multiple time-lapse measurements at Jakobshavn Isbræ in 2004, 2007 and 2010 even allowed for the documentation of the migration of the glacier's grounding line.
Recognition of subglacial drainage channels. Another example of the possibility to obtain information about subglacial processes from vertical motion fields derived from terrestrial image sequences is the detection of a subglacial channel during a glacier lake outburst flood (GLOF) event. During a GLOF event, a glacier margin lake spontaneously starts to drain underneath the glacier, causing flash floods in downstream valleys. The event is triggered by the opening of a subglacial channel that drains the lake water and is quickly widened by erosion and melting. A typical GLOF event may take a few hours to days. After the outburst, the channel soon collapses and the lake refills until a new channel opens up and the cycle starts again (see Clague and Evans, 1994; Tweed and Russell, 1999; Dussaillant et al., 2010).

A time-lapse camera observing the water level of glacier margin lakes may be used as a GLOF early warning system if sudden water level changes can be detected reliably in the image data by a suitable image processing procedure (Mulsow et al., 2014). Vertical motion patterns of the glacier surface obtained from the same image data may depict a suitable basis to localize such a subglacial channel and to investigate the development of the channel during and after a GLOF event. As an example, in Schwalbe et al. (2016) a case study is presented where the high temporal resolution of the motion fields of the Colonia Glacier allow for an analysis of the tunnel formation and collapse in relation to the changing water level of the lake Cachet II obtained from a GLOF early warning camera. By an integration of daily subsidence fields of the glacier over a period of several days, the drainage channel could be localized and visualized for several GLOF events at Cachet II. 


\section{Conclusions}

The presented approach allows for the analysis of monoscopic image sequences in order to derive glacier motion vector fields with high spatial and temporal resolution based on tracking a large number of glacier surface points. These motion vector fields are of great value for the analysis of glacier motion behaviour, particularly of glaciers showing high motion dynamics. Terrestrial photogrammetric time-lapse image measurements are a powerful tool for providing glacier motion data with high accuracy at a high spatial and temporal resolution. Compared to other measuring methods, which are used for the observation of glaciers, automatic image sequence analysis also offers the advantages of a high flexibility and versatility at rather low instrumental cost. Obviously, there is the disadvantage that terrestrial measurement methods are not suitable for investigations of large areas. They are therefore to be considered as complementary methods to measurement methods based on aircraft or satellite imagery, which have the potential to cover large areas but at a limited temporal resolution.

The presented methodology is a holistic approach which consists of the determination of glacier surface point motion vectors and trajectories in image space and the transformation of the motion vectors into object space. Thereby, the reliability and accuracy of the resulting trajectories can be refined applying specific solutions to solve various problems which are typical for outdoor motion analysis. The main influences, which distort measurements in image space, are migrating shadows on the glacier surface, instabilities of the camera and the oblique view of the terrestrial camera. The influence of the shadows can be reduced during image point tracking by a technique of iteratively excluding shadowinfluenced pixels from image matching. The influence of camera movement can be modelled and compensated for by tracking fixed targets visible in the image sequences and applying a RANSAC-based approach to robustly determine parameters describing the effects of camera motion. The tracking on surfaces recorded with oblique camera view benefits from rectangular patches which are individually adapted to the distance range covered by the patch. The scaling and georeferencing of the trajectories are mostly influenced by the quality of the available digital elevation model (DEM) and the accuracy of the time-lapse camera's registration to the DEM. To prevent possible registration errors, a simultaneous generation of the DEM and the time-lapse image orientation in a bundle adjustment of a photogrammetric network is advantageous and also provides a solution even if no external DEM of the object is available. Furthermore, during the process of geo-referencing diverse practical aspects need to be considered. Thus, we proposed different possible measurement set-ups, which can be chosen depending on whether the focus of the measurement is on high positioning accuracy or light-weight measurement equipment and flexibility in the field.
The error analyses of the trajectories derived from the image sequences showed that the individual motion values can be determined with accuracy in the order of several centimetres for glacier surface points at a distance of several kilometres from the camera. The method allows for the measurement of both horizontal and vertical motion components. The described practical examples have shown that terrestrial time-lapse measurements can provide a suitable data basis for a wide spectrum of applications in glaciology.

While the method of image sequence analysis as described here is optimized for the application for the motion analysis of glaciers, the use of the approaches is also conceivable for manifold other environmental investigations, e.g. motion analysis of landslides or even flow measurements of rivers.

Data availability. The developed software for glacier surface point tracking and geo-referencing is available at https://tu-dresden. de/geo/emt/.

Competing interests. The authors declare that they have no conflict of interest.

Special issue statement. This article is part of the special issue "4-D reconstruction of earth surface processes: multi-temporal and multi-spatial high-resolution topography". It is not associated with a conference.

Acknowledgements. The work presented in this paper was supported by the German Research Foundation (MA 2504/5-1, MA 2504/14-1), by the German Federal Ministry of Education and Research (BMBF International Office) and by the support program "Support the best" (StB) of the TU Dresden.

Edited by: Damià Vericat

Reviewed by: three anonymous referees

\section{References}

Ackermann, F.: Digital image correlation: Performance and potential application in photogrammetry, Photogramm. Rec., 11, 429439, https://doi.org/10.1111/j.1477-9730.1984.tb00505.x, 1984.

Ahn, Y. and Box, J. E.: Instruments and methods: ice velocities from time-lapse photos: technique development and first results from Extreme Ice Survey (EIS) in Greenland, J. Glaciol., 56, 723-734, https://doi.org/10.3189/002214310793146313, 2010.

Brown, L. G.: A Survey of Image Registration Techniques, ACM Comput. Surv., 24, 325-376, https://doi.org/10.1145/146370.146374, 1992.

Clague, J. J. and Evans, S. E.: Formation and failure of natural dams in the Canadian Cordillera, Geological Survey of Canada Bulletin, 464, 35 pp., 1994.

Danielson, B. and Sharp, M.: Development and application of a time-lapse photograph analysis method to investigate the link 
between tidewater glacier flow variations and supraglacial lake drainage events, J. Glaciol., 59, 287-302, 2013.

Delaunay, B. N.: Sur la sphère vide, Bulletin of Academy of Sciences of the USSR, 7, 793-800, 1934.

Dietrich, R., Maas, H.-G., Bässler, M., Rülke, A., Richter, A., Schwalbe, E., and Westfeld, P.: Jakobshavn Isbræ, West Greenland: Flow velocities and tidal interaction of the front area from 2004 field observations, J. Geophys. Res., 112, F03S21, https://doi.org/10.1029/2006JF000601, 2007.

Dussaillant, A., Benito, G., Buytaert, W., Carling, P., Meier, C., and Espinoza, F.: Repeated glacial-lake outburst floods in Patagonia: an increasing hazard?, Nat. Hazards, 54, 469-481, 2010.

Eiken, T. and Sund, M.: Photogrammetric methods applied to Svalbard glaciers: accuracies and challenges, Polar Res., 31, 18671, https://doi.org/10.3402/polar.v31i0.18671, 2012.

Eltner, A. and Schneider, D.: Analysis of Different Methods for 3D Reconstruction of Natural Surfaces from Parallel-Axes UAV Images, Photogramm. Rec., 30, 279-299, 2015.

Finsterwalder, R.: Geschwindigkeitsmessungen an Gletschern mittels Photogrammetrie, Zeitschrift für Gletscherkunde, 29, 251262, 1931.

Fischler, M. A. and Bolles, R. C.: Random sample consensus: a paradigm for model fitting with applications to image analysis and automated cartography, ACM, 24, 381-395, https://doi.org/10.1145/358669.358692, 1981.

Flotron, A.: Photogrammetrische Messung von Gletscherbewegungen mit automatischer Kamera, Photogrammetric measurements of glacier movements with automatic cameras, Vermessung, Photogrammetrie und Kulturtechnik, 71, 15-17, 1973.

Förstner, W.: On the geometric precision of digital correlation. In: International Archives of Photogrammetry, Proceedings of the Symposium of the ISPRS Commission III, Helsinki, 24, 176189,1982

Grün, A.: Adaptive Least Squares Correlation: A Powerful Image Matching Technique, South African Journal of Photogrammetry, Remote Sensing \& Cartography, 14, 175-187, 1985.

Harrison, W. D., Raymond, C. F., and MacKeith, P.: Short period motion events on Variegated Glacier as observed by automatic photography and seismic methods, Ann. Glaciol., 8, 8289, https://doi.org/10.1017/S0260305500001191, 1986.

James, M. R. and Robson, S.: Sequential digital elevation models of active lava flows from ground-based stereo time-lapse imagery, ISPRS J. Photogramm., 97, 160-170, 2014.

James, M. R., How, P., and Wynn, P. M.: Pointcatcher software: analysis of glacial time-lapse photography and integration with multitemporal digital elevation models, J. Glaciol., 62, 159-169, https://doi.org/10.1017/jog.2016.27, 2016.

James, M. R., Robson, S., d'Oleire-Oltmanns, S., and Niethammer, U.: Optimising UAV topographic surveys processed with structure-from-motion: ground control quality, quantity and bundle adjustment, Geomorphology, 280, 51-66, 2017.

Kramer, N. and Wohl, E.: Estimating fluvial wood discharge using time-lapse photography with varying sampling intervals, Earth Surf. Proc. Land., 39, 844-852, https://doi.org/10.1002/esp.3540, 2014.

Kraus, K.: Photogrammetry - Geometry from Images and Laser Scans, 2nd Edn., 2014, de Gruyter, Berlin, 2007.

Krimmel, R. M. and Rasmussen, L. A.: Using sequential photography to estimate ice velocity at the terminus of Columbia Glacier, Alaska, Ann. Glaciol., 8, 117-123, https://doi.org/10.1017/S0260305500001270, 1986.

Lewis, J. P.: Fast normalized cross-correlation, Vision Interface, 10, 120-123, 1995.

Luhmann, T., Robson, S., Kyle, S., and Harley, I.: Close Range Photogrammetry: Principles, Methods and Applications, Book, 1st Edn., Whittles, UK, 229-266, 2006.

Maas, H.-G., Dietrich, R., Schwalbe, E., Bäßler, M., and Westfeld, P.: Analyse des Bewegungsverhaltens des Jakobshavn Isbræ Gletschers in Grönland durch monokulare Bildsequenzanalyse, Photogramm. Fernerkun., 2, 93-102, 2006.

Matsuoka N.: Combining Time-Lapse Photography and Multisensor Monitoring to Understand Frost Creep Dynamics in the Japanese Alps, Permafrost Periglac. Process., 25, 94-106, https://doi.org/10.1002/ppp.1806, 2014.

Messerli, A. and Grinsted, A.: Image georectification and feature tracking toolbox: ImGRAFT, Geosci. Instrum. Method. Data Syst., 4, 23-34, https://doi.org/10.5194/gi-4-23-2015, 2015.

Mulsow, C., Koschitzki, R., and Maas, H.-G.: Photogrammetric monitoring of glacier margin lakes. In: Geomatics, Natural Hazards and Risk, 6, 600-613, https://doi.org/10.1080/19475705.2014.939232, 2014.

Nichols, M. H., Nearing, M., Hernandez, M., and Polyakov V. O.: Monitoring channel head erosion processes in response to an artificially induced abrupt base level change using time-lapse photography, Geomorphology, 265, 107-116, 2016.

Niemeier, W.: Ausgleichungsrechnung, de Gruyter Verlag, Berlin, New York, ISBN 3-11-014080-2, 2002.

Parajka, J., Haas, P., Kirnbauer, R., Jansa, J., and Blöschl, G.: Potential of time-lapse photography of snow for hydrological purposes at the small catchment scale, Hydrol. Process., 26, 3327-3337, https://doi.org/10.1002/hyp.8389, 2012.

Pillewizer, W.: Photogrammetrische Gletscherforschung, Bildmessung und Luftbildwesen, 2, 66-73, 1938.

Rivera, A., Corripio, J., Bravo, C., and Cisternas, S.: Glaciar Jorge Montt dynamics derived from photos obtained by fixed cameras and satellite image feature tracking, Ann. Glaciol., 53, 147-155, https://doi.org/10.3189/2012AoG60A152, 2012.

Rosenau, R., Schwalbe, E., Dietrich, R., and Maas, H.-G.: Grounding line migration and high resolution calving dynamic of Jakobshavn Isbræ, West Greenland, J. Geophys. Res.-Earth, 118, 382395, https://doi.org/10.1029/2012JF002515, 2013.

Schneider, D.: Geometrische und stochastische Modelle für die integrierte Auswertung terrestrischer Laserscannerdaten und photogrammetrischer Bilddaten, $\mathrm{PhD}$ thesis, Technische Universität Dresden, 2008.

Schwalbe, E., Maas, H-.G., Dietrich, R., and Ewert, H.: Glacier velocity determination from multi-temporal terrestrial long range laserscanner point clouds, in: IAPRS, 37, Proceedings of the 21. Congress of ISPRS, Beijing, China, 457-462, 2008.

Schwalbe, E., Koschitzki, R., and Maas, H.-G.: RECOGNITION OF DRAINAGE TUNNELS DURING GLACIER LAKE OUTBURST EVENTS FROM TERRESTRIAL IMAGE SEQUENCES, Int. Arch. Photogramm. Remote Sens. Spatial Inf Sci., XLI-B8, 537-543, https://doi.org/10.5194/isprs-archivesXLI-B8-537-2016, 2016.

Schwalbe, E., Kröhnert, M., Koschitzki, R., Cárdenas, C., and Maas, H-G.: Proceedings of the First IEEE International Symposium of Geoscience and Remote Sensing (GRSS- 
CHILE) 2017, 17066477, https://doi.org/10.1109/GRSSCHILE.2017.7996022, 2017.

Stumpf, A., Augereau, E., and Delacourt C.: Photogrammetric discharge monitoring of small tropical mountain rivers: A case study at Rivière des Pluies, Réunion Island, Water Resour. Res., 52, 4550-4570, https://doi.org/10.1002/2015WR018292, 2016.

Taylor, J. R.: Introduction To Error Analysis: The Study of Uncertainties in Physical Measurements, University Science Books, 2nd Edn., 1997.

Travelletti, J., Delacourt, C., Allemand, P., Malet, J.-P., Schmittbuhl, J., Toussaint, R., and Bastard, M.: Correlation of multitemporal ground-based optical images for landslide monitoring: Application, potential and limitations, ISPRS J. Photogramm., 70, 39-55, https://doi.org/10.1016/j.isprsjprs.2012.03.007, 2012.
Tweed, F. S. and Russell, A. J.: Controls on the formation and sudden drainage of glacier-impounded lakes: implications for jökulhlaup characteristics, Prog. Phys. Geogr., 23, 79-110, 1999.

Walter, T. R.: Low cost volcano deformation monitoring: optical strain measurement and application to Mount St. Helens data, Geophys. J. Int., 186, 699-705, https://doi.org/10.1111/j.1365246X.2011.05051.x, 2011.

Zitová, B. and Flusser, J.: Image registration methods: a survey, Image Vision Comput., 21, 977-1000, 2003. 